\title{
Ability of artificial intelligence to detect T1 esophageal squamous cell carcinoma from endoscopic videos: supportive effects of real-time assistance
}

\section{Sho Shiroma}

Japanese Foundation For Cancer Research

Toshiyuki Yoshio ( $\square$ toshiyuki.yoshio@jfcr.or.jp )

Japanese Foundation For Cancer Research

Yusuke Kato

Al Medical Service inc

Yoshimasa Horie

Toho University Ohashi Medical Center

Ken Namikawa

Japanese Foundation For Cancer Research

Yoshitaka Tokai

Japanese Foundation For Cancer Research

Shoichi Yoshimizu

Japanese Foundation For Cancer Research

Yusuke Horiuchi

Japanese Foundation For Cancer Research

Akiyoshi Ishiyama

Japanese Foundation For Cancer Research

Toshiaki Hirasawa

Japanese Foundation For Cancer Research

Tomohiro Tsuchida

Japanese Foundation For Cancer Research

Naoki Akazawa

National Center For Global Health and Medicine

Junichi Akiyama

National Center For Global Health and Medicine

Tomohiro Tada

Al Medical Service inc

Junko Fujisaki

Japanese Foundation For Cancer Research 


\section{Research Article}

Keywords: esophageal cancer, detection, diagnosis, endoscopy, artificial intelligence, convolutional neural networks

Posted Date: December 21st, 2020

DOI: https://doi.org/10.21203/rs.3.rs-126656/v1

License: (c) (1) This work is licensed under a Creative Commons Attribution 4.0 International License. Read Full License

Version of Record: A version of this preprint was published at Scientific Reports on April 8th, 2021. See the published version at https://doi.org/10.1038/s41598-021-87405-6. 


\section{Abstract}

Diagnosis using artificial intelligence (Al) with deep learning could be useful in endoscopic examinations. We investigated the ability of Al to detect superficial esophageal squamous cell carcinoma (ESCC) from esophagogastroduodenoscopy (EGD) videos. We retrospectively collected 8428 EGD images of esophageal cancer to develop a convolutional neural network through deep learning. We evaluated the detection accuracy of the Al diagnosing system compared with that of 18 endoscopists. We used 144 EGD videos for the two validation sets. First, we used 64 EGD observation videos of ESCC using both white light imaging (WLI) and narrow-band imaging (NBI). We then evaluated the system using 80 EGD videos from 40 patients (20 with superficial ESCC and 20 with non-ESCC). In the first set, the Al system correctly diagnosed $100 \%$ ESCCs. In the second set, it correctly detected $85 \%(17 / 20)$ ESCCs. Of these, $75 \%(15 / 20)$ and $55 \%(11 / 22)$ were detected by WLI and NBI, and the positive predictive value was $36.7 \%$. The endoscopists correctly detected $45 \%$ (25-70\%) ESCCs. With Al real-time assistance, the sensitivities of the endoscopists were significantly improved without Al assistance $(p<0.05)$. Al can detect superficial ESCC from EGD videos with high sensitivity and improve endoscopists' detection of ESCC with real-time support.

\section{Introduction}

Esophageal cancer is the sixth most common cause of mortality worldwide, accounting for almost 508,000 deaths annually ${ }^{[1,2]}$ Esophageal squamous cell carcinoma (ESCC) is the most common histological type of esophageal cancer throughout Asia, specifically Japan ${ }^{[3,4]}$. ESCC diagnosed in advanced stages often requires invasive treatment and has a poor prognosis; therefore, early detection is important for optimal prognosis ${ }^{[4]}$. However, early diagnosis remains difficult, and early-stage disease can be overlooked during endoscopic examination.

It can be challenging to correctly diagnose ESCC at early stages using only white light imaging (WLI). lodine staining can improve ESCC detection with high sensitivity and specificity; however, it can cause severe discomfort and increases the procedure time ${ }^{[5,6,7]}$. It is therefore used only for high-risk patients. Narrow-band imaging (NBI) is a revolutionary technology of optical image-enhanced endoscopy that facilitates ESCC detection without iodine staining ${ }^{[8-10]}$. NBI is easier to use than iodine staining and does not cause patient discomfort. However, NBI has insufficient sensitivity (53\%) for detecting ESCC when used by inexperienced endoscopists ${ }^{[11]}$. Therefore, there is an urgent and unmet need to improve ESCC detection for less experienced practitioners.

Computer-aided diagnosis using artificial intelligence (Al) with deep learning methods could be a useful adjunct to endoscopic examination that could improve detection of early cancers ${ }^{[12-14]}$. Our group was the first to report good diagnostic performance of Al using deep learning to detect esophageal cancer, including ESCC and adenocarcinoma, from still endoscopic images. In our study, Al had a sensitivity of $98 \%$ and could distinguish superficial and advanced cancer with an accuracy of $98 \%{ }^{[12]}$. In superficial 
cancers, the Al diagnosing system could differentiate pathological mucosal and submucosal microinvasive (SM1) cancers from submucosal deep invasive (SM2) cancers; this can help determine the appropriate treatment course for each patient ${ }^{[15]}$.

In this study, we evaluated the ability of Al to detect ESCC from esophagogastroduodenoscopy (EGD) videos. Analyzing still images, Al can evaluate only a limited area, however, numerous images are required to screen the entire esophagus, which requires a lot of time. In EGD videos, the whole esophagus can be evaluated without taking pictures of the non-cancerous areas. To ensure that Al would detect ESCC in a fast-moving situation, for example, in the event that an inexperienced endoscopists examines the esophagus to quickly and does not notice the lesions, we prepared two validation video sets which include slow-speed and high-speed video sets. Analysis of Al diagnosis using videos will aid in realizing the real-time support of Al diagnosing systems for endoscopic examination.

\section{Methods}

This study was approved by the Institutional Review Board of the Cancer Institute Hospital (No. 20161171) and the Japan Medical Association (ID JMA-II A00283). Informed consent or an acceptable substitute was obtained from all patients.

\section{Preparation of training image sets and construction of a convolutional neural network (CNN) algorithm}

For this single-center retrospective study, we obtained EGD images taken from February 2016 to April 2017 at the Cancer Institute Hospital, Tokyo, Japan, as described previously ${ }^{[12]}$. Briefly, we collected 8428 training images of esophageal lesions histologically confirmed to be ESCC or adenocarcinoma. The training esophageal cancer images included 397 lesions of ESCC, including of 332 superficial cancers and 65 advanced cancers. Training images included 6026 and 2402 images obtained using WLI and NBI endoscopy, respectively. Poor-quality images resulting from halation, blur, defocus, mucus, and poor air insufflation were excluded. Magnified images obtained by magnifying endoscopy were also excluded. All images of esophageal cancer lesions were manually marked by a well-experienced endoscopist. These images were used to develop a deep learning algorithm using an Al diagnosing system for esophageal cancer.

To develop our Al-based diagnosing system, we used a deep neural network architecture (https://arxiv.org/abs/1512.02325), referred to as a "Single Shot MultiBox Detector" [12].

\section{Al system to detection ESCC in videos}

The Al diagnosing system recognized 30 continuous frames of still images in $1 \mathrm{sec}$ of video and detected ESCC in the same manner as the analysis of still images. When the Al detected a cancer, it reviewed the video for $0.5 \mathrm{sec}$ ( 15 frames). If the reviewed section included a cancer image in more than 3 frames, and the maximum interval from the latest cancer image was $0.1 \mathrm{sec}$ ( 3 frames), the Al diagnosed the lesion as cancer, giving a discovery signal (Figure 1a). This setting was based on a small number of videos that 
were independent of the validation dataset and obtained in a preliminary examination. The Al diagnosing system inserted the image of the recognized cancer on the left side of the monitor (Figure 1b, c, d), indicating that it diagnosed the lesion as cancerous. If the inserted image included any part of ESCC, we considered it positive, and if the inserted image did not include ESCC, we considered it a false-positive result. Endoscopists could easily verify whether the Al diagnosing system had correctly detected the cancer.

\section{Validation EGD video set and Al diagnosis}

The performance of the Al diagnosing system was evaluated using independent validation EGD videos. We used a total of 144 EGD videos for the two validation sets. As a slow-speed video validation set, we prepared a dataset of 64 videos of 32 ESCC patients obtained using both WLI and NBI from August 2018 to August 2019 at the Cancer Institute Hospital. In the EGD videos, ESCC was observed while the endoscope was moving slowly. The whole lesions were observed for 5 to $15 \mathrm{sec}$. When the Al diagnosing system recognized a cancer, it indicated it with a bordering square and inserted the image on the left side of the monitor. Because all videos included cancer, we examined only sensitivity in this validation set.

As a high-speed video validation set, we prepared a dataset of 80 videos of WLI and NBI endoscopies performed for 40 patients from August 2018 to August 2019 at the Cancer Institute Hospital. The dataset included 20 patients with 22 superficial ESCC lesions and 20 patients without ESCC. We used EGD videos inserting the endoscope from the cervical esophagus to the esophagogastric junction (EGJ) at a speed of $2 \mathrm{~cm} / \mathrm{sec}$ without stopping or focusing on the specific lesions. These EGD videos were considered the speed at which the endoscopist taking the video passed by without noticing the lesion in routine examination.

Sensitivity, specificity, positive predictive value (PPV), and negative predictive value (NPV) of the Al diagnosing system to detect ESCC from each EGD video were calculated as follows: sensitivity, number of EGD videos in which the Al diagnosing system correctly diagnosed cancer divided by the total number of EGD videos with cancer; specificity, number of EGD videos in which the Al diagnosing system determined that no cancerous lesion existed divided by the total number of videos without cancer; PPV, number of EGD videos in which the AI diagnosing system accurately detected cancer divided by the total number of videos in which the Al diagnosing system detected cancer; and NPV, number of EGD videos in which the Al diagnosing system accurately determined that no cancerous lesion existed divided by the total number of videos that the Al diagnosing system determined as not having a cancerous lesion. In a comprehensive analysis, when the AI diagnosing system detected ESCC in either WLI or NBI videos, we defined this as a correct diagnosis.

\section{Comparison with endoscopists}

We prepared two sets of validation videos for diagnosis by endoscopists. One set was composed of the same set of high-speed videos that the Al diagnosed. The other set was the same as the first set but included the diagnostic real-time assistance of $\mathrm{Al}$, with the $\mathrm{Al}$ indicating cancers with a rectangular border 
without inserting the image on the left side of the monitor. In this video set, we examined the additive effect of the Al system to the diagnostic ability of the endoscopists. These validation video sets were diagnosed by 18 endoscopists, including 7 board-certified endoscopists and 11 non-certified endoscopists, at the Japan Gastroenterological Endoscopy Society. Endoscopists watched high-speed videos on a personal computer and pushed a button when they detected ESCC (correct answer). However, the answer was considered incorrect when an endoscopist failed to pushed the button and did not recognize the ESCC in the video. Moreover, if endoscopists noticed the lesion but could not confirm that is was ESCC while the lesion was on the monitor, the answer was considered incorrect. These rules were strictly adhered to, in order to ensure the accuracy of this analysis. The endoscopists could push the button as many times as they detected ESCC. Each endoscopist diagnosed one set of videos chosen randomly. After one month of washout, the endoscopists diagnosed the other validation video set. Between the two rounds of analysis, the endoscopists were not given feedback on their performance or the correct answers.

\section{Statistical analysis}

All continuous variables are expressed as median and range. The differences in Al sensitivity and specificity by WLI and NBI were compared using McNemar's test.

The sensitivities of the endoscopists with or without Al assistance were compared using the MannWhitney test with GraphPad Prism software (GraphPad Software, Inc, La Jolla, CA, USA). A p value of $<0.05$ was considered statistically significant.

\section{Results}

\section{Al diagnosis of the slow-speed validation video set}

The characteristics of patients and lesions in the validation video set are summarized in Table $1 \mathrm{a}$. In the set, there were more men than women, the median age was 67.5 years, and half the lesions were located in the middle thoracic esophagus. These characteristics are typical for ESCC in the Japanese population

[16]. The median tumor size was $17 \mathrm{~mm}$, and most lesions were mucosal ESCC (T1a) (Table 1a). Therefore, the sensitivity of the Al diagnosing system was $100 \%$ for both WLI (32/32) and NBI (32/32).

\section{Al diagnosis of the high-speed validation video set}

In the high-speed validation set, $90 \%$ patients were men, and the median age was 70 years. The median tumor size was $17 \mathrm{~mm}$ with $95 \%$ being T1a and $5 \%$ being T1b (Table $1 \mathrm{~b}$ ). The sensitivity of the Al diagnosing system was $85 \%$ (17/20) for the comprehensive diagnosis, whereas the sensitivities based on WLI (Supplementary Video S1) and NBI (Supplementary Video S2) were 75\% and 55\%, respectively (Figure 2). The specificity in NBI was significantly higher than that in WLI $(80 \%$ vs $30 \%, p<0.01)$ (Table 2$)$.

\section{Causes of false-positive and false-negative results in the high-speed video set}


The most frequent cause of false-positive results (Table 3 ) was a shadow in the esophageal lumen (Figure $3 a$ ), which accounted for $41 \%$ of all false-positives. Normal structures and benign lesions, such as the EGJ (Figure 3b), post-endoscopic resection scars (Figure 3c), and mucosal inflammation (Figure 3d) were also misdiagnosed as cancer.

With regard to false-negative results (Table 3 ), nearly half the false-negative images were due to esophageal inflammation in the background mucosa (Figure 4a). Other common causes were anterior wall lesions (Figure 4b) and obscure ESCC lesions, particularly with WLI endoscopy (Figure 4c), which were sometimes difficult to diagnose even by expert endoscopists. The Al diagnosing system also missed a lesion measuring $5 \mathrm{~mm}$ in diameter (Figure 4d), which was the smallest lesion in the EGD videos. Endoscopists could detect it in the shape of a submucosal tumor-like elevated lesion.

\section{Outcomes of endoscopists in the high-speed validation video set}

The median sensitivity of the endoscopists for the comprehensive cancer diagnosis was $45 \%$ (range, 25$70 \%$ ), whereas the median sensitivities based on WLI and NBI videos were $25 \%$ (range, $15-45 \%$ ) and $35 \%$ (range, 15-60\%), respectively (Figure 5). There was no difference in sensitivity between board-certified endoscopists and non-certified endoscopists.

With Al real-time assistance indicating cancers with a rectangle, the sensitivities of the endoscopists were significantly improved relative to their sensitivities without Al assistance $(p<0.05)$. The sensitivities were improved in 13 of 18 endoscopists by a median of $10 \%(5-25 \%)$.

\section{Discussion}

We evaluated the computer-aided detection of ESCC from EGD videos that employed Al-based CNN with deep learning. Al diagnosis of ESCC in videos has been reported recently in other studies ${ }^{[17,18]}$. In the videos of these studies, endoscopists carefully observed the ESCC lesions and diagnosed them using Al. The videos were similar to the slow-speed video sets in our study, and the results were good. However, it is impossible to observe the whole esophagus carefully for every patient in routine screening examination, as this would require an extended amount of time. As endoscopists are required to detect the lesions first during routine screening examinations, we also tested high-speed video sets. Furthermore, we have shown that the diagnosis of cancers by the endoscopists improved with Al assistance.

We first evaluated slow-speed videos and achieved a detection rate of $100 \%$ in both WLI and NBI. We then examined the Al's performance using high-speed validation video. The sensitivity of the Al diagnosing system in high-speed videos was $85 \%$, which was much higher than the $45 \%$ sensitivity of endoscopists. Their sensitivity was significantly improved to $52.5 \%$ with Al assistance.

From a point of view, videos are very different from still images. However, Al recognizes $1 \mathrm{sec}$ of video as a sequence of 30 frames of still images and detects ESCC in the same manner as still images of the same quality. In the slow-speed videos, the Al detection rate was $100 \%$, which is consistent with previous 
reports ${ }^{[17]}$. Although this is an important step in using Al to diagnose cancer, these results are not sufficient to prove that $\mathrm{Al}$ is useful for detecting cancers that humans overlook. To address this in a clinical situation, we used a high-speed validation video set. The sensitivity of the Al diagnosing system was $85 \%$ in this high-speed video set. The difference between these two results can be explained by fewer focused clear images in high-speed videos, including more unclear bridging images in between clear images, because the endoscope moved continuously without stopping or focusing on any lesion. It was difficult to detect ESCCs that the scope passed in all consecutive frames in the high-speed videos. It was also more challenging to detect ESCCs during peristalsis, when the ESCC appeared bent or shrunk on the moving esophageal wall, because we only trained the Al system on well-extended esophageal walls. To improve these weaknesses, training videos for an Al system should include plenty of bridging images to achieve higher robustness.

Although the sensitivity of NBI was low and the sensitivity of WLI was slightly high, there was no significant difference between the two observation method. The number of cases was not large in this study; however, the sensitivity of NBI was sufficiently high with regard to slow-speed validation set and still image evaluation in previous studies ${ }^{[12]}$. In addition, it was still higher than the reported PPV of endoscopists examining NBI endoscopies (45\% for experienced endoscopists and 35\% for less experienced endoscopists) ${ }^{[11]}$. In daily clinical practice, false-positive results for cancer screening are considered more acceptable than false-negative results. Adding magnifying endoscopy reportedly improves PPV ${ }^{[18-19]}$. However, we believe that the Al system without magnifying endoscopy presented here would be most useful for primary detection in clinics or hospitals without well-experienced endoscopists on staff, so we specifically aimed to develop a non-magnifying system in this study.

We also analyzed causes of false-positive and false-negative results. False-positives were often caused by shadows of the esophageal lumen and EGJ (Table 3), similar to our still image analysis ${ }^{[12]}$. Nearly half false-negative results were due to inflammation of the background mucosa, which can also be difficult for endoscopists to differentiate. The second most common reason for false-negatives was anterior wall lesions, which can be difficult for endoscopists to detect on tangential views.

The sensitivity of the Al was better than that of 15 endoscopists using the same videos. The sensitivity of diagnosis by the endoscopists was $45 \%$, demonstrating the difficulty of obtaining a proper diagnosis. Moreover, this result suggests that Al could identify 40\% ESCCs that were missed by the endoscopists. We hypothesize that the low sensitivity of endoscopists was due to the increased speed of the videos and strict criteria of correct answers in which endoscopists had to diagnose the lesions. However, Al could diagnose ESCC in fast-moving situations that were difficult for endoscopists. Furthermore, we showed the improved diagnostic ability of the endoscopists with Al assistance.

Endoscopists usually move the endoscope quickly through the esophagus, as in the high-speed validation videos, until a suspected cancerous lesion is noticed. They confirm the presence of cancer by observation under magnification or biopsy, although many point out cancer by non-magnifying observation. After detecting the lesion, endoscopists can diagnose it as ESCC by examining a slow-speed 
video set and still images. Al may help clinicians detect these cancers in real time. After identifying a lesion, the endoscopist should stop to examine it more carefully, as in the slow-speed validation videos. The image of the ESCC that appears on the monitor will let the clinician know that the Al has identified the suspected lesion as cancerous. Diagnostic assistance using the Al system would be helpful in both slow-moving and fast-moving situations.

This study has several limitations. First, this was a single-center, retrospective study. However, we think the results are reliable because the ESCC diagnosis was objectively verified. Second, we moved the endoscope at several speeds to imitate a screening endoscopy; however, we saw an outcome at only two speeds. The Al detected 100\% ESCCs in the slow-speed videos that imitated careful lesion observation. In the high-speed videos that imitated endoscopy without careful lesion inspection. Third, we validated a limited number of ESCCs, but we believe that our previous analysis of still images compensates for the lack of variety in cancers in this study.

\section{Conclusion}

The Al-based diagnostic system demonstrated a high diagnostic accuracy to detect ESCC from EGD videos. Moreover, the detection of endoscopists were improved by the real-time assistance of $\mathrm{Al}$ diagnosing system in high-speed videos. Next, we plan to demonstrate that the Al diagnosing system would be helpful to detect ESCCs in a clinical study. We hope that the Al-based diagnostic system presented here will improve ESCC detection and facilitate earlier diagnosis in daily clinical practice in the near future.

\section{Declarations}

\section{Data availability}

The datasets analysed during the current study are available from the corresponding author on reasonable request.

\section{Acknowledgments}

This study was supported by grants from the Uehara Memorial Foundation, Takeda Science Foundation, and Daiwa Securities Health Foundation and a JSPS KAKENHI Grant (Number 19K08408).

\section{Author contributions}

All authors contributed to the study conception and design. Material preparation, data collection and analysis

were performed by Sho Shiroma and Toshiyuki Yoshio. The first draft of the manuscript was written by Sho 
Shiroma and all authors commented on previous versions of the manuscript. All authors read and approved the

final manuscript.

\section{Additional information}

\section{Competing interests}

Tomohiro Tada is a shareholder in Al Medical Service, Inc. Sho Shiroma, Toshiyuki Yoshio, Yusuke Kato, Yoshimasa Horie, Ken Namikawa, Yoshitaka Tokai, Shoichi Yoshimizu, Yusuke Horiuchi, Akiyoshi Ishiyama, Toshiaki Hirasawa, Tomohiro Tsuchida, Naoki Akazawa, Junichi Akiyama, and Junko Fujisaki declare no potential conflict of interest.

\section{Human rights statement and informed consent}

All procedures followed were in accordance with the ethical standards of the responsible committee on human experimentation (institutional and national) and with the Helsinki Declaration of 1964 and later versions. Informed consent or substitute for it was obtained from all patients for being included in the study.

\section{References}

1. Ferlay, J. et al. Estimating the global cancer incidence and mortality in 2018: GLOBOCAN sources and methods. Int. J. Cancer.144, 1941-1953 (2019).

2. Agrawal, N. et al. Comparative genomic analysis of esophageal adenocarcinoma and squamous cell carcinoma. Cancer Discov.2, 899-905 (2012).

3. Enzinger, P. C. \& Mayer, R. J. Esophageal cancer. N. Engl. J. Med. 349, 2241-2252 (2003).

4. Kuwano, H. et al. Guidelines for Diagnosis and Treatment of Carcinoma of the Esophagus April 2012 edited by the Japan Esophageal Society. Esophagus.12, 1-30 (2015).

5. Fagundes, R. B. et al. Occult dysplasia is disclosed by Lugol chromoendoscopy in alcoholics at high risk for squamous cell carcinoma of the esophagus. Endoscopy.31, 281-285 (1999).

6. Kameyama, H. et al. The efficacy and diagnostic significance of sodium thiosulphate solution spraying after iodine dyeing of the esophagus. Dig. Endosc.6, 181-186 (1994).

7. Kondo, H. et al. Sodium thiosulfate solution spray for relief of irritation caused by Lugol's stain in chromoendoscopy. Gastrointest. Endosc.53, 199-202 (2001).

8. Nagami, Y. et al. Usefulness of non-magnifying narrow-band imaging in screening of early esophageal squamous cell carcinoma: a prospective comparative study using propensity score matching. Am. J. Gastroenterol.109, 845-854 (2014).

9. Lee, Y. C. et al. Transnasal endoscopy with narrow-band imaging and Lugol staining to screen patients with head and neck cancer whose condition limits oral intubation with standard endoscope 
(with video). Gastrointest. Endosc.69, 408-417 (2009).

10. Kuraoka, K. et al. Early esophageal cancer can be detected by screening endoscopy assisted with narrow-band imaging (NBI). Hepatogastroenterology.56, 63-66 (2009).

11. Ishihara, R. et al. Prospective evaluation of narrow-band imaging endoscopy for screening of esophageal squamous mucosal high-grade neoplasia in experienced and less experienced endoscopists. Dis. Esophagus.23, 480-486 (2010).

12. Horie, Y. et al. Diagnostic outcomes of esophageal cancer by artificial intelligence using convolutional neural networks. Gastrointest. Endosc.89, 25-32 (2019).

13. Tamashiro, A. et al. Artificial-intelligence-based detection of pharyngeal cancer using convolutional neural networks. Dig Endosc.32, 1057-1065 (2020).

14. Hirasawa, T. et al. Application of artificial intelligence using a convolutional neural network for detecting gastric cancer in endoscopic images. Gastric Cancer.21, 653-660 (2018).

15. Tokai, Y. et al. Application of artificial intelligence using convolutional neural networks in determining the invasion depth of esophageal squamous cell carcinoma. Esophagus.17, 250-256 (2020).

16. Japanese Esophageal Society. Japanese Classification of Esophageal Cancer, 11th Edition: part I. Esophagus.14, 1-36 (2017).

17. Guo, L. et al. Real-time automated diagnosis of precancerous lesions and early esophageal squamous cell carcinoma using a deep learning model (with videos). Gastrointest. Endosc.91, 41-51 (2020).

18. Fukuda, H. et al. Comparison of performances of artificial intelligence versus expert endoscopists for real-time assisted diagnosis of esophageal squamous cell carcinoma (with video). Gastrointest. Endosc.92, 848-855 (2020).

19. 19. Ohmori, M. et al. Endoscopic detection and differentiation of esophageal lesions using a deep neural network. Gastrointest.91, 301-309.e1 (2020).

\section{Tables}

Table 1: Characteristics of the validation video sets

a) The slow-speed validation video sets 


\begin{tabular}{|ll|}
\hline Patient characteristics $(\mathrm{n}=32)$ & \\
\hline Sex $(\mathrm{n}),($ Male/Female) & $30 / 2$ \\
\hline Age (years), [median(range)] & $67.5(48-84)$ \\
\hline Lesion characteristics (n=32) & \\
\hline Tumor size (mm), [median(range)] & $17(5-52)$ \\
\hline Depth of tumor (n), (EP/LPM/MM/SM) & $7 / 21 / 3 / 1$ \\
\hline Macroscopic type (n), (0-I/0-Ila/0-Ilb/0-Ilc) & $1 / 2 / 13 / 16$ \\
\hline Part of esophagus (n)m (Ce/Ut/Mt/Lt) & $1 / 4 / 16 / 11$ \\
\hline Region (n), (anterior/posterior/left/right) & $9 / 6 / 7 / 10$ \\
\hline
\end{tabular}

b) The high-speed validation video sets

\begin{tabular}{|ll|}
\hline Patient characteristics ( $\mathrm{n}=40)$ & \\
\hline Sex (n), (Male/Female) & $36 / 4$ \\
\hline Age (years), [median(range)] & $70(57-83)$ \\
\hline Lesion characteristics ( $\mathrm{n}=22)$ & \\
\hline Tumor size (mm), [median(range)] & $17(5-60)$ \\
\hline Depth of tumor (n), (EP/LPM/MM/SM) & $8 / 10 / 3 / 1$ \\
\hline Macroscopic type (n), (0-I/0-Ila/0-IIb/0-IIc) & $0 / 2 / 4 / 16$ \\
\hline Part of esophagus (n), (Ce/Ut/Mt/Lt) & $0 / 6 / 11 / 5$ \\
\hline Region (n), (anterior/posterior/left/right) & $8 / 4 / 8 / 2$ \\
\hline
\end{tabular}

EP: epithelium, LPM: lamina propria, MM: muscularis mucosae, SM: submucosa Ce: cervical esophagus, Ut: upper thoracic esophagus, Mt: middle thoracic esophagus, Lt: lower thoracic esophagus

Table 2: Detailed results of the Al-based diagnosis for each case

\begin{tabular}{|lllll|}
\hline & Sensitivity & Specificity & PPV & NPV \\
WLI & $75 \%$ & $30 \%$ & $52 \%$ & $55 \%$ \\
\hline & $(15 / 20)$ & $(6 / 20)$ & $(15 / 29)$ & $(6 / 11)$ \\
NBI & $55 \%$ & $80 \%$ & $73 \%$ & $64 \%$ \\
\hline & $(11 / 20)$ & $(16 / 20)$ & $(11 / 15)$ & $(16 / 25)$ \\
\hline
\end{tabular}


PPV: positive predictive value, NPV: negative predictive value, Al: artificial intelligence, WLI: white light imaging, NBI: narrow-band imaging

Table 3: Details of the false-positive and false-negative lesions

\begin{tabular}{|ll|}
\hline False-positive results $(\mathrm{n}=74)$ & \\
\hline Shadow of lumen & $41 \%(30)$ \\
\hline Inflammation & $32 \%(24)$ \\
\hline Post-ER scar & $9 \%(7)$ \\
\hline EGJ & $18 \%(13)$ \\
\hline False-negative results $(\mathrm{n}=12)$ * & \\
\hline Inflammation of background mucosa & $42 \%(5)$ \\
\hline Anterior wall lesion & $33 \%(4)$ \\
\hline Obscure ESCC by WLI & $17 \%(2)$ \\
\hline Less than 5 mm in size & $8 \%(1)$ \\
\hline
\end{tabular}

* 12 lesions in 11 cases

ER: endoscopic resection, EGJ: esophagogastric junction, ESCC: esophageal squamous cell carcinoma, WLI: white light imaging

\section{Figures}



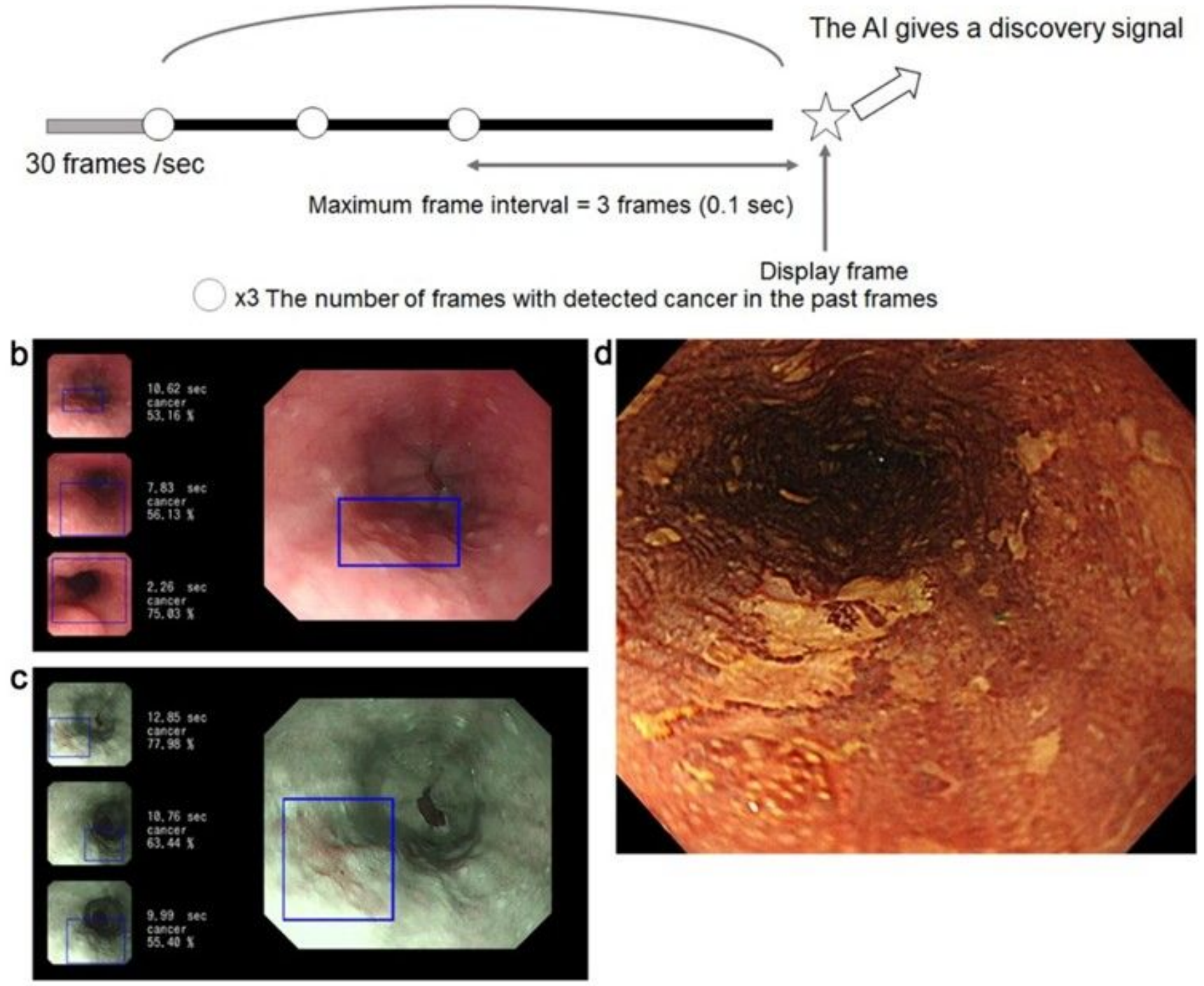

\section{Figure 1}

System of Al diagnosis in endoscopic videos and representative images of Al detection of ESCC (a) When the Al detected a cancerous lesion, the Al reviewed the video for $0.5 \mathrm{sec}$ ( 15 frames). If the reviewed section of video included a cancer image in more than 3 frames and the maximum interval from the latest cancer image was $0.1 \mathrm{sec}$ ( 3 frames), the Al diagnosed the lesion as cancer, giving a discovery signal. (b, c) When the Al recognized a cancerous lesion, a frame was displayed in the endoscopic image surrounding the lesion of interest. The Al inserted the image of the recognized cancer on the left side of the monitor, indicating that it diagnosed the lesion as cancerous. (d)When the Al diagnosed cancer matched the iodine unstained area which was pathologically diagnosed as ESCC, the Al was considered correct. Abbreviations - Al: artificial intelligence, ESCC: esophageal squamous cell carcinoma 
(\%)

\section{Sensitivity of Al diagnosis for each case}

90

85

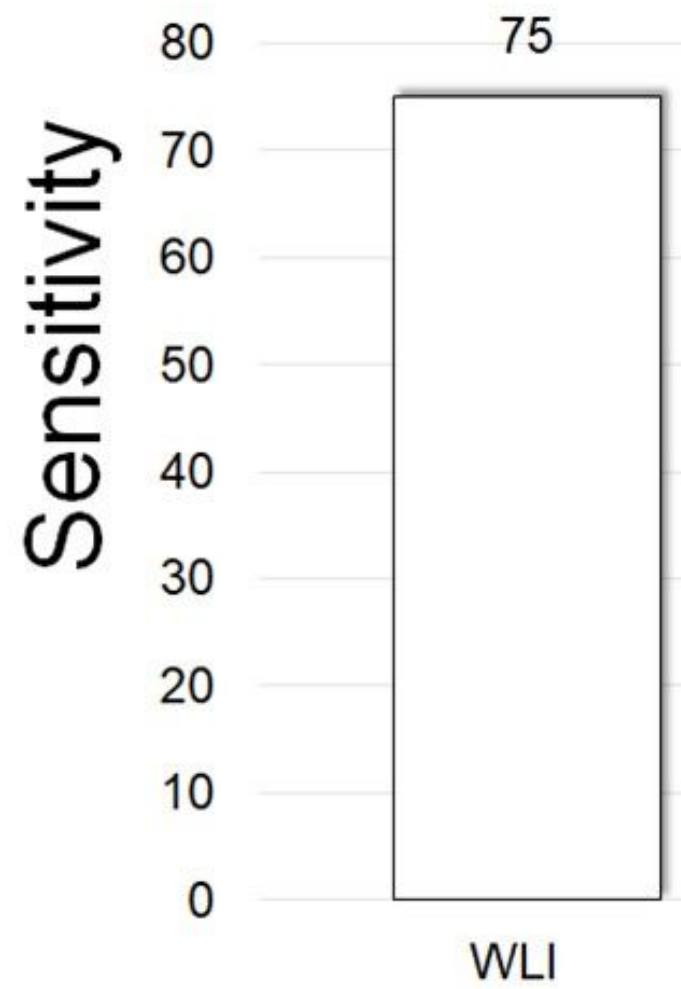

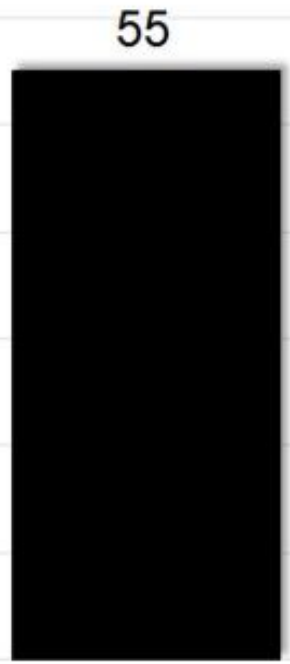

$\mathrm{NBI}$

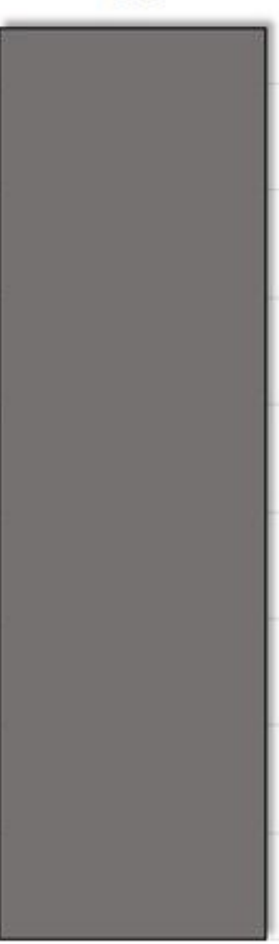

$\mathrm{WLI}+\mathrm{NBI} *$

\section{Figure 2}

Sensitivity of the Al diagnosis for each case The sensitivity of the Al diagnosis for each case was slightly higher in WLI than in NBI, but not significantly. ${ }^{*} \mathrm{WLI}+\mathrm{NBI}$ : when a cancer was diagnosed with either WLI or $\mathrm{NBI}$, we considered that the Al had detected the cancer. Abbreviations - Al: artificial intelligence, WLI: white light imaging, NBI: narrow-band imaging 


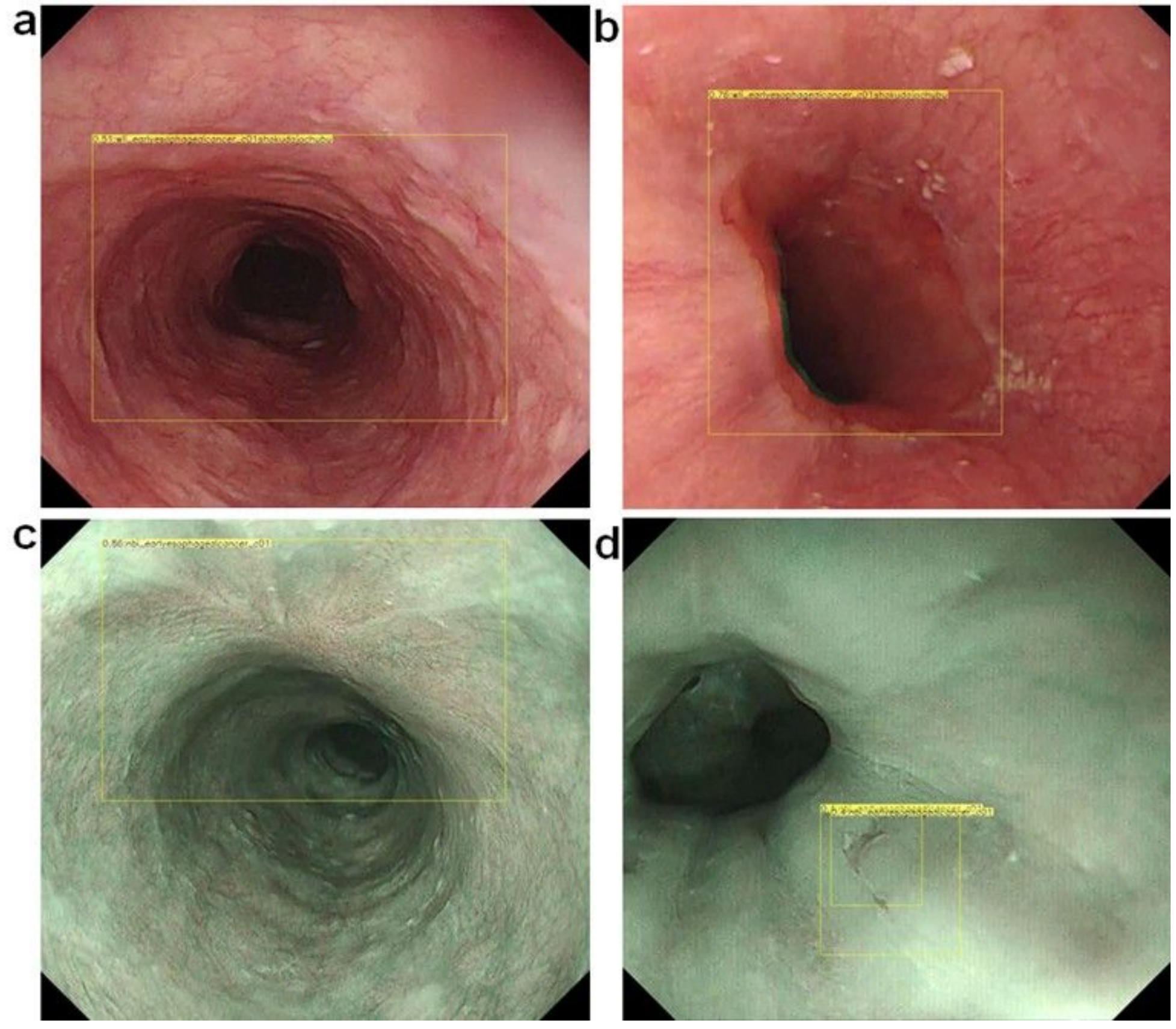

Figure 3

Examples of false-positive images The yellow squares indicate areas that were misdiagnosed as cancer. a: shadow of lumen, b: EGJ, c: post ER scar, d: Inflammation. Abbreviations - ER: endoscopic resection, EGJ: esophagogastric junction 

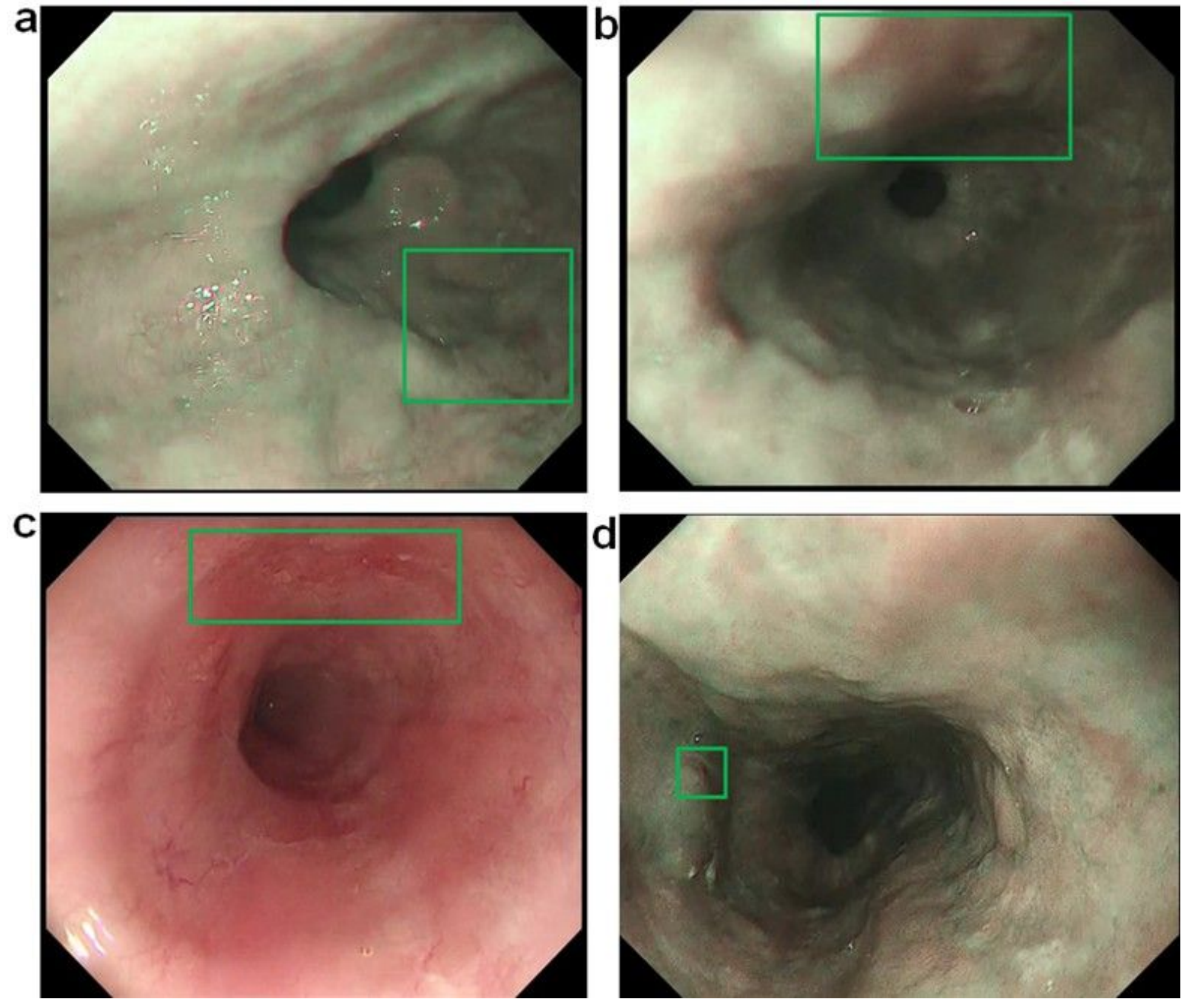

Figure 4

Examples of false-negative images The cancers in the shown images were missed for the following estimated causes. a: inflammation of background mucosa, b: anterior wall lesion, c: obscure ESCC by WLI, d: ESCC less than $5 \mathrm{~mm}$. Abbreviations - ESCC: esophageal squamous cell carcinoma, WLI: white light imaging 


\section{Sensitivity of human endoscopists diagnosis for each case}

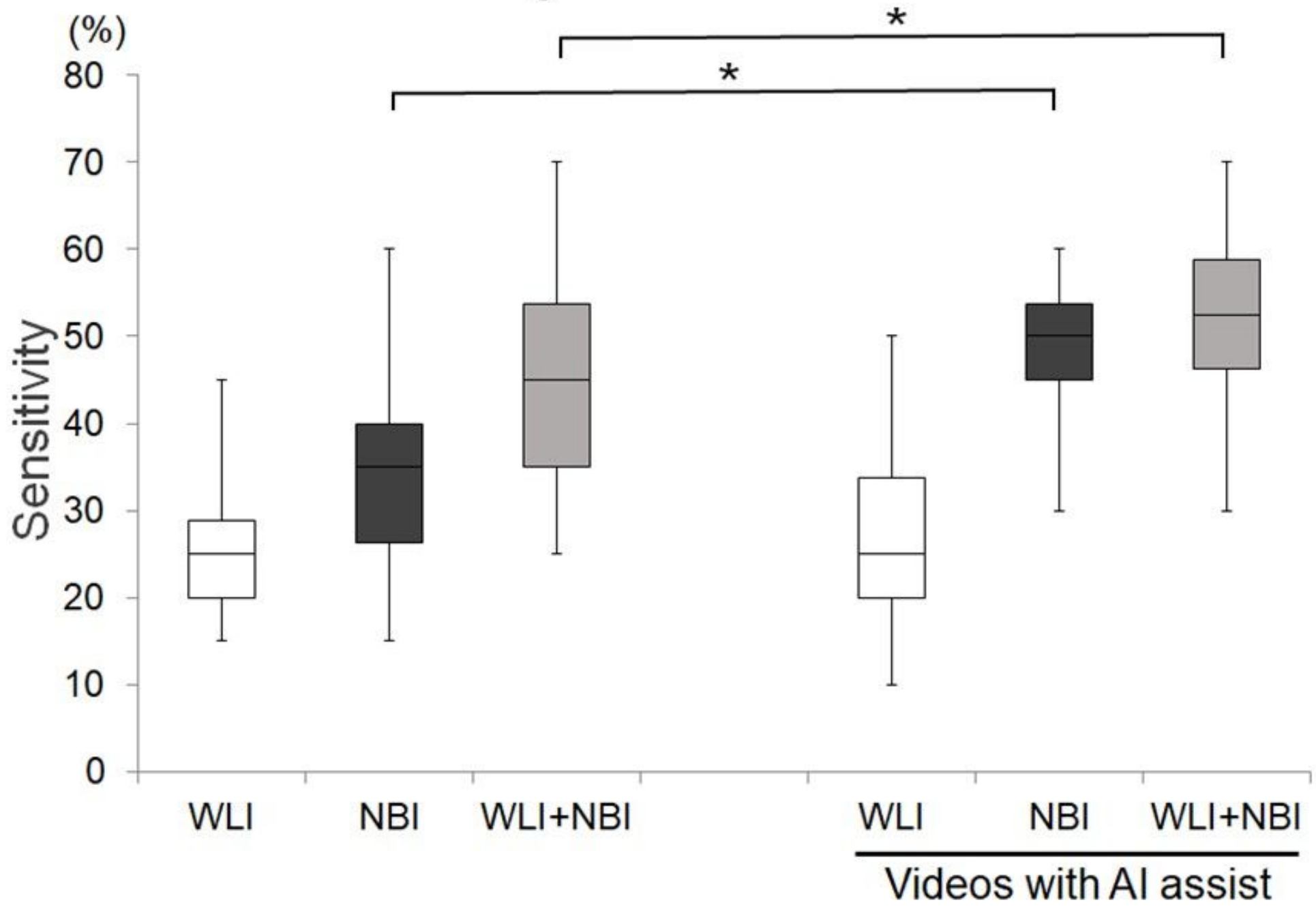

Figure 5

Sensitivity of the endoscopists for each case The median sensitivity is represented by the center line in the box, which indicates the IQR. The range is indicated by whiskers. When diagnosed with either WLI or $\mathrm{NBI}$, we considered the endoscopists to have detected the cancers (WLI + NBI). Abbreviations - IQR: interquartile range, WLI: white light imaging, NBI: narrow-band imaging $* P<0.05$

\section{Supplementary Files}

This is a list of supplementary files associated with this preprint. Click to download.

- VideoSupplementaryvideoS1.

- VideoSupplementaryvideoS2. 